\title{
DIVERSIDADE E ESTRUTURA GENÉTICA ESPACIAL EM DUAS POPULAÇÕES DE Myracrodruon urundeuva Fr. All. SOB DIFERENTES CONDIÇÕES ANTRÓPICAS ${ }^{1}$
}

\author{
Mario Luiz Teixeira de Moraes ${ }^{2}$, Paulo Yoshio Kageyama ${ }^{3}$ e Alexandre Magno Sebbenn ${ }^{4}$
}

\begin{abstract}
RESUMO - O objetivo deste trabalho foi estudar, por locos isoenzimáticos, a diversidade e a estrutura genética espacial de genótipos de Myracrodruon urundeuva em duas populações naturais, uma no Sudoeste (SelvíriaSEL) e outra no Sudeste (Paulo de Faria-PFA) do Brasil. Para isso, foram avaliados cinco sistemas isoenzimáticos, 25 e 30 indivíduos adultos das populações SEL e PFA, respectivamente. A estimativa da divergência genética entre populações foi baixa $(0,043)$. As heterozigosidades observada e esperada foram altas nas populações (0,317 e 0,511, respectivamente), e o excesso significativo de heterozigotos foi detectado na população PFA $(-0,252)$. A análise da distribuição genética espacial dos genótipos a partir do índice $I$ de Moran revelou estruturação significativa até $5.224 \mathrm{~m}$ na população mais explorada $(\mathrm{SEL}=0,09)$ e tendência à distribuição aleatória na população menos explorada ( $\mathrm{PFA}=-0,02$ ). A provável causa da estruturação na população SEL foi a dispersão de sementes próxima às árvores-matriz, associada ao processo de recolonização a partir de sementes oriundas de poucos genótipos remanescentes. As implicações dos resultados são discutidas do ponto de vista da conservação e do melhoramento genético.
\end{abstract}

Palavras-chave: Espécies arbóreas, índice $I$ de Moran, diversidade genética e isoenzimas.

\section{DIVERSITY AND SPATIAL GENETIC STRUCTURE IN TWO POPULATIONS OF Myracrodruon urundeuva Fr. All. UNDER DIFFERENT ANTROPIC CONDITIONS}

\begin{abstract}
The diversity and spatial genetic distribution of Myracrodruon urundeuva genotypes were studied in two Brazilian populations in the Southwest (Selvíria-SEL) and Southeast Brazilian regions (Paulo de FariaPFA). Twenty-five and thirty adult individuals were evaluated for five allozyme systems in SEL and PFA populations, respectively. Estimates of the genetic divergence between populations were low (0.043). Observed and expected heterozygosities were high in both populations ( 0.317 to 0.511 , respectively). Significant and excessive number of heterozygotes was detected in PFA population (-0.252). The spatial distribution analysis through Moran's index $\mathrm{I}$ revealed a significant structuring up to 5,224 $\mathrm{m}$ in the more exploited population $(S E L=0.09)$ and a trend to randomness in the less exploited area $(P F A=-0.02)$. Seed dispersion near mother trees and the recolonization process through seeds from few genotypes are probable causes of the structuring in the $S E L$ population. The implications of the results are discussed from the conservation and breeding point of views.
\end{abstract}

Key words: Forest tree species, I Moran index, genetic diversity and allozymes.

\footnotetext{
${ }^{1}$ Recebido em 16.06.2003 e aceito para publicação em 25.11.2004.

${ }^{2}$ FEIS/UNESP, Av. Brasil Centro, 56, 15385-000 Ilha Solteira-SP.

${ }^{3}$ ESALQ/USP, Departamento de Ciências Florestais, Av. Pádua Dias, 11, 13418-900 Piracicaba-SP.

${ }^{4}$ Instituto Florestal de São Paulo, Caixa Postal 1322, 01059-970 São Paulo-SP.
} 


\section{INTRODUÇÃO}

Myracrodruon urundeuva (Anacardiaceae) ou aroeira é uma espécie arbórea tropical secundária tardia de ampla distribuição geográfica, ocorrendo de $3^{03} 0^{\prime} S$ (Brasil) a $25^{\circ} \mathrm{S}$ (Argentina), sendo também encontrada na Bolívia e no Paraguai. A espécie ocorre em várias regiões fitoecológicas de $17 \mathrm{~m}$ a $1.200 \mathrm{~m}$ de altitude: Floresta Estacional Semidecidual, Floresta Estacional Decidual, Cerrado e Cerradão, Caatinga, Chaco SulMato-Grossense e Pantanal Mato Grossense (CARVALHO, 1994). M. urundeuva é considerada espécie dióica (SANTIN e LEITÃO FILHO, 1991), mas há relatos de monoicia (NOGUEIRA et al., 1982) e ocorrência de hermafroditismo juntamente com dioicia (CARVALHO, 1994). Os frutos são dispersos por anemocoria (CARVALHO, 1994), e a polinização é feita por abelhas.

A reprodução por cruzamentos, a ampla distribuição geográfica e a capacidade de adaptar-se a diversos ecossistemas evidenciam que $M$. urundeuva apresenta altos níveis de variabilidade genética. Contudo, no passado, a espécie foi muito explorada devido às qualidades e utilidades de sua madeira para construção civil e indústria moveleira (CARVALHO, 1994). Atualmente, $M$. urundeuva é pouco encontrada na região centro-sul do Brasil, especialmente no Estado de São Paulo, onde não é mais possível encontrá-la em grandes proporções em seu estado silvestre, a não ser em reservas estaduais e pequenas reservas particulares. Nestas, às vezes, observa-se que a distribuição é agrupada em forma de manchas, formadas por menos de uma centena de indivíduos. As manchas podem ser constituídas pela dispersão das sementes próximas às árvores-matriz, o que favorece a estruturação genética das populações.

A maior parte da variação genética encontra-se dentro de populações $(97,26 \%)$ e apenas $2,74 \%$, entre populações (MORAES, 1992). Desse modo, a estrutura genética dentro de populações de aroeira passa a ter fundamental importância, pois se refere à distribuição não-aleatória dos genótipos, formando manchas, onde as frequiências alélicas são mais homogêneas do que a esperada pela pressuposição de dispersão aleatória de genótipos. A estrutura genética espacial dentro de populações de plantas é primariamente determinada pelos efeitos da limitada dispersão de pólen e sementes, isolamento em pequenas manchas, mortalidade diferencial e seleção para micro-“habitats”. A estrutura genética espacial influencia a dinâmica da endogamia biparental, depressão por endogamia e a operação da seleção natural (EPPERSON, 1993). Intuitivamente, em espécies arbóreas podem-se associar distribuição espacial, parentesco e probabilidade de cruzamento, sobretudo em espécies polinizadas por animais, em que existe a tendência de indivíduos próximos se intercruzarem mais do que indivíduos amplamente espaçados. Se estes forem aparentados, devido à dispersão de sementes próximas às árvores-matriz, a endogamia na população pode aumentar (COLES e FOWLER, 1976). O parentesco de uma geração, quando medido em termos de coancestria, corresponde à endogamia que pode ser gerada na descendência, se os indivíduos forem cruzados.

O principal tema deste artigo é a distribuição espacial de genótipos de $M$. urundeuva dentro de duas populações com diferentes históricos de antropização: Selvíria, MS, no sudoeste, e Paulo de Faria, SP no Sudeste brasileiro. O objetivo foi avaliar a diversidade e a distribuição espacial de genótipos de $M$. urundeuva em duas populações, sob diferentes condições antrópicas, para estudos de conservação e melhoramento de populações.

\section{MATERIAL E MÉTODOS}

O estudo foi realizado em duas populações de M. urundeuva, Selvíria, MS (SEL), e Paulo de Faria, SP sob diferentes condições antrópicas. Essas populações foram escolhidas por serem remanescentes da exploração, com diferentes intensidades, da floresta primária. A distância entre as populações é de aproximadamente $250 \mathrm{~km}$. As árvores onde foram feitas as coletas de sementes foram georreferenciadas com o auxílio de GPS. A primeira população localiza-se no município de Selvíria, MS (SEL), onde foram coletadas sementes de polinização livre em 25 árvores amplamente espaçadas (distância média $=1.000 \mathrm{~m}$ ), localizadas em área de pastagem sob forte degradação antrópica. Estimase que a área da população seja de aproximadamente 10.000 ha. A população foi altamente explorada, ocupando atualmente extensa área de pastagem. A segunda população localiza-se na Estação Ecológica do Instituto Florestal de São Paulo, no município de Paulo de Faria, SP (PFA), onde foram coletadas sementes de 30 árvores (distância média $=50 \mathrm{~m}$ ). Essa população foi pouco explorada e ocupa pequena área (aproximadamente 50 ha) dentro da Estação Ecológica de Paulo de Faria (435,73 ha), onde, no passado, ocorreu extração de alguns 
exemplares da espécie. Embora não existam levantamentos fitossociológicos nessas áreas, relatando a densidade de indivíduos por hectare, é possível observar que M. urundeuva encontra-se em baixa densidade em ambas as populações ( 1 a 5 indivíduos por hectare).

As sementes foram utilizadas para determinar o genótipo materno, devido à dificuldade de revelação de número suficiente de locos isoenzimáticos que permitisse a avaliação adequada dos genótipos adultos. O genótipo das árvores maternas foi inferido a partir da segregação de suas progênies (20 progênies por planta materna, totalizando 500 plântulas em SEL e 600 em PFA), com base no genótipo parental mais provável, segundo o método de Brown e Allard (1970), utilizando o programa MLTR (RITLAND, 1997).

Tecidos foliares de aproximadamente 20 plântulas por progênie foram avaliados por eletroforese de isoenzimas no Laboratório de Genética de Populações e Silvicultura do Departamento de Fitotecnia, Tecnologia de Alimentos e Socioeconomia da FEIS/UNESP, Ilha Solteira, SP. As enzimas foram extraídas de tecidos foliares, empregando-se aproximadamente $20 \mathrm{mg}$ de tecido de limbo foliar, $10 \mathrm{mg}$ de areia lavada, $7 \mathrm{mg}$ de Polivinil Pirrolidona-40 (PVP 40), 7 mg de Polivinil Pirrolidona60 (PVP-60) e 200 microlitros da solução de extração número 1, de Alfenas (1998). Aeletroforese foi a horizontal, conduzida em gel de amido de milho (penetrose 30) a $12 \%$, usando-se o tampão eletrodo-gel tris citrato, $\mathrm{pH} 8,5$. As corridas foram realizadas em geladeira à temperatura de $5^{\circ} \mathrm{C}$, em corrente constante. Foram avaliados cinco sistemas isoenzimáticos: aspartato aminotransferase (AAT), leucina aminopeptidase (LAP), fosfoglucose isomerase (PGI), 6-fosfogluconato desidrogenase (6PGDH) e fosfoglucomutase (PGM). O protocolo de revelação das isoenzimas foi o mesmo descrito por Alfenas (1998). Os locos foram designados seqüencialmente com o alelo de maior migração anodal denominado 1, o próximo 2, e assim por diante. As enzimas selecionadas expressaram fenótipos consistentes em estrutura de subunidades e interpretação genética de outros estudos em plantas, como documentado por Wendel e Weeden (1989) e Alfenas (1998), apesar de a base genética dos locos não ter sido testada por cruzamentos controlados.

A diversidade genética intrapopulacional foi analisada pelo número médio de alelos por locos $(\hat{A}$, obtido pela divisão do número total de alelos pelo número de locos), porcentagem de locos polimórficos ( $\hat{P}$, obtido pela divisão do número de locos polimórficos pelo número total de locos), sendo um loco considerado polimórfico quando a freqüência do alelo mais comum não ultrapassava $95 \%$, heterozigosidade observada ( $\hat{H}_{o}=1-\sum \hat{P}_{i i}$, em que: $\hat{P}_{i i}=$ freqüência dos genótipos homozigotos) e diversidade gênica esperada em equilíbrio de Hardy-Weinberg $\left(\hat{H}_{e}=1-\sum \hat{p}_{i}^{2}\right.$, em que: $p_{i}=$ freqüência alélica estimada do $i$-ésimo alelo), estimados a partir do programa BIOSYS-1 (SWOFFORD; SELANDER, 1989). Também, foi estimado o índice de fixação não viesado $(\hat{F})$, com base em Weir (1996):

$$
\hat{F}=\frac{\left(\hat{H}_{e}-\hat{H}_{0}\right)+\frac{2}{2 n} \hat{H}_{o}}{\hat{H}_{e}-\frac{1}{2 n} \hat{H}_{o}}
$$

A distribuição da variabilidade genética entre e dentro das populações foi calculada pelo coeficiente de coancestralidade $\left(\hat{\theta}_{p}\right)$, obtido por análise da variância de freqüências gênicas, segundo Weir (1996). A significância estatística dos valores médios de $\hat{F}$ e $\hat{\theta}_{p}$ foi calculada pelo intervalo de confiança a $95 \%$ de probabilidade, utilizando-se 10.000 reamostragens bootstrap sobre locos e usando o programa GDA, de Lewis e Zaykin (1999).

A distribuição espacial dos genótipos foi analisada por autocorrelação espacial, empregando o índice $I$, de Moran. Para análise de autocorrelação espacial, os dados de cada genótipo foram codificados para suas frequiências alélicas, ou seja, quando o genótipo era homozigoto, no alelo sob análise recebeu valor 1,0 , quando heterozigoto recebeu valor 0,5 e quando homozigoto para outro alelo recebeu valor 0,0 (SOKAL e ODEN, 1978). Se o loco era dialélico, a análise era feita em apenas um dos alelos, já que o segundo contribui com informação idêntica. Quando o loco tinha mais de dois alelos, a análise foi realizada em todos os alelos. Em cada população foram criadas cinco classes de distâncias, devido ao pequeno tamanho amostral (n) das populações (25 genótipos em SEL e 30 em PFA). Maior número de classes implicaria menor número de pares possíveis de comparações de genótipos dentro de cada classe [ $n(n-1) / 2 \times 5]$. Portanto, menor poder para testar a significância estatística do índice. Todos os possíveis pares de combinações de genótipos foram considerados como um conjunto e acessados para cada uma das classes de distância. A extensão dessas classes foi selecionada, equalizando-se o número total de cada

R. Árvore, Viçosa-MG, v.29, n.2, p.281-289, 2005 
conjunto de pares de comparações, a fim de se obter maior precisão nas estimativas. Foram calculados os valores do índice $I$, de Moran (SOKAL e ODEN, 1978), de cada uma das classes de distância, em cada loco, e a média dos locos pela expressão-padrão:

$$
\hat{I}=n \sum_{i} \sum_{j} w_{i j} Z_{i} Z_{j} / w \sum_{i} Z_{i}^{2}
$$

em que: $\mathrm{w}_{\mathrm{ij}}=1$ em todas as células $i$ e $j$ localizadas em uma classe de distância $(r, r+\Delta r)$, e do contrário, $\mathrm{w}_{\mathrm{ij}}=0 ; W$ é a soma de todos os pesos (contagem de todos os pares usados em todos os casos); e é o desvio da frequiência alélica da $i$-ésima célula da média. O valor esperado de $\mathrm{I}=-1 /(n-1)$ aproxima-se de zero em grandes $n$. Os valores estimados do índice $I$ de Moran foram usados para testar a significância dos desvios dos valores esperados sobre a hipótese de nulidade de distribuição aleatória (CLIFFe ORD, 1981). A significância total de cada correlograma foi testada, usando-se critérios de Bonferroni (SAKAI e ODEN, 1983). As análises de autocorrelação espacial e os testes foram realizados empregando-se o programa SAAP (versão 4.3), de D. Wartenberg (WARTENBERG, 1989).

\section{RESULTADOS E DISCUSSÃO}

Os cinco sistemas isoenzimáticos revelaram seis locos, sendo cinco polimórficos, em ambas as populações, segregando de dois a três alelos, e um monomórfico (Pgm1) na população PFA. O mesmo número médio de alelos por loco $(\hat{A}=2,33)$ foi encontrado nas duas populações, com a ressalva de que a população PFA apresentou maior porcentagem de locos polimórficos ( $\hat{P}=0,830)$, heterozigosidade observada $\left(\hat{H}_{0}=0,511\right) \mathrm{e}$ esperada em equilíbrio de Hardy-Weinberg $\left(\hat{H}_{e}=0,408\right)$ (Tabela 1). Em espécies como M. urundeuva, que se

Tabela 1 - Índices de diversidade genética em árvores adultas de duas populações de M. urundeuva

Table 1 - Indexes of genetic diversity in adult trees of two populations of Myracrodruon urundeuva

\begin{tabular}{lcccccc}
\hline População & $\boldsymbol{n}$ & $\hat{A}$ & $\hat{P}_{(\mathbf{9 5} \%)}$ & $\hat{H}_{o}$ & $\hat{H}_{e}$ & $\hat{F}$ \\
\hline Selvíria-SEL & 25 & 2,33 & 40 & 0,333 & 0,317 & $-0,053$ \\
Paulo de & 30 & 2,33 & 83 & 0,511 & 0,408 & $-0,258^{*}$ \\
Faria-PFA & & & & & & \\
\hline
\end{tabular}

*: $P \leq 5 \%$; A significância do índice $\hat{F}$ foi estimada pelo intervalo de confiança a $95 \%$ de probabilidade, a partir de 10.000 reamostragens bootstrap. $n=$ número de árvores amostradas; $\hat{A}=$ número médio de alelos por loco; $\hat{P}=$ porcentagem de locos polimórficos; $\hat{H}_{o}=$ heterozigosidade observada; $\hat{H}_{e}=$ heterozigosidade esperada; e $\hat{F}=$ índice de fixação. reproduzem, predominantemente, por cruzamentos, ocupam grandes extensões geográficas e diferentes "habitats", espera-se que apresentem altos níveis de variabilidade genética (HAMRICK e LOVELESS, 1986). Comparando-se as heterozigosidades esperadas com as calculadas por Hamrick e Godt (1990) para a média das espécies de cruzamento e polinização entomófila $(0,167)$, dispersão de sementes anemocórica $(0,144)$, espécies amplamente dispersas $(0,202)$, tropicais $(0,148)$ e arbóreas $(0,177)$, verifica-se que as populações de M. urundeuva apresentam valores muito superiores a esses. Outras espécies arbóreas tropicais dióicas também têm revelado altos níveis de heterozigosidade: 0,182 em Genipa americana L. (SEBBENN et al., 1998), 0,450 em Rhus trichocarpa M. (CHUNG et al., 1999) e 0,277 em Eurya japonica T. (CHUNG e EPPERSON, 2000), embora inferiores aos detectados em M. urundeuva.

A divergência genética entre as populações $\left(\hat{\theta}_{p}\right)$ foi baixa, 0,043 (0,002 a 0,100), mas estatisticamente diferente de zero. A distribuição da diversidade genética entre e dentro de populações, em locos neutros, como a maioria das isoenzimas estudadas em plantas, é determinada pelo sistema de reprodução, deriva genética e fluxo gênico. Espécies alógamas, polinizadas por animais e dispersas pelo vento, como M. urundeuva, geralmente apresentam baixos níveis de divergência genética entre populações (HAMRICK e LOVELESS, 1986). A baixa divergência genética entre as populações indica que o fluxo gênico é alto entre as populações e que os polinizadores e a dispersão de sementes pelo vento seriam capazes de transpor a longa distância que as separa (aproximadamente $250 \mathrm{~km}$ ). Entretanto, atualmente estas se encontram isoladas por culturas agrícolas, aglomerados urbanos, estradas etc., e a baixa divergência reflete, provavelmente, o fluxo gênico ocorrido no passado, quando as florestas eram contínuas ou ocupavam grande parte da extensão que as separaram e não o fluxo atual. O isolamento, possivelmente, no futuro reflitirá em aumento na diferenciação entre populações por deriva genética.

A análise da distribuição espacial dos genótipos, a partir do índice I, de Moran, revelou forte estruturação na população SEL e fraca na população PFA (Tabelas 2 e 3 e Figura 1). Na população SEL, foram encontrados nove valores de I Moran (22,5\% dos valores) significativamente diferentes do esperado $(-0,042)$, sendo quatro positivos na classe 1 , dois negativos na classe 2 , um negativo na classe 3 e dois negativos na classe 
5 (Tabela 2). Para o correlograma total, quatro dos 10 casos (alelos) foram significativos, reforçando a hipótese de estruturação. Indícios de estruturação são observados também na estimativa média do índice $I$, de Moran, por classe de distância (Tabela 2 e Figura 1), em que $I$ assume valores positivos nas três primeiras classes e negativos nas duas últimas, apesar de nenhum valor médio ser estatisticamente diferente do esperado. $\mathrm{Na}$ população PFA, foram observados apenas quatro valores de $I$, de Moran ( $8,8 \%$ dos valores), estatisticamente diferente do esperado $(-0,034)$, sendo um valor positivo na primeira classe de distância e três negativos nas classes 2, 3 e 4 (Tabela 3). O correlograma total dos alelos não foi significativo para nenhum dos valores, e os valores médios do índice $I$, de Moran, foram todos baixos, negativos e não-significativos, reforçando a hipótese de ausência de estruturação. A forte estruturação na população SEL e ausência na PFA devem estar associada ao fato de a primeira população ter sido formada a partir da regeneração após a exploração, enquanto a população PFA foi pouco explorada. A regeneração a partir de sementes de poucos indivíduos (gargalo genético), combinada com a disponibilização de espaço para recolonização (área explorada), pode ser a explicação para a mais forte estruturação detectada na população SEL. Resultado semelhante, no que se refere à perturbação antrópica na estruturação de populações de aroeira, foi encontrado por Lacerda e Kageyama (2003) no semiárido brasileiro.

Tabela 2 - Índices I de Moran para classes de distâncias na população SEL de M. urundeuva Table 2 - Indexes of Moran I for classes of distances in SEL population of Myracrodruon urundeuva

\begin{tabular}{|c|c|c|c|c|c|c|c|}
\hline \multirow[b]{2}{*}{ Loco } & \multirow[b]{2}{*}{ Alelo } & \multicolumn{5}{|c|}{ Classes de Distância (Número de Pares) } & \multirow[b]{2}{*}{$P$} \\
\hline & & $5.224 \mathrm{~m} \mathrm{(60)}$ & $8.810 \mathrm{~m} \mathrm{(60)}$ & $11.830 \mathrm{~m} \mathrm{(60)}$ & $14.795 \mathrm{~m} \mathrm{(60)}$ & $19.654 \mathrm{~m}(60)$ & \\
\hline \multirow[t]{3}{*}{ Aat 1} & 1 & $0,30 * *$ & $-0,16$ & $-0,11$ & $-0,05$ & $-0,19$ & 0,006 \\
\hline & 2 & $0,29 * *$ & $-0,17$ & $-0,15$ & $-0,00$ & $-0,19$ & 0,006 \\
\hline & 3 & 0,01 & $-0,13 * *$ & $-0,10$ & 0,01 & 0,01 & 0,033 \\
\hline Aat2 & 1 & $-0,01$ & $-0,16$ & $-0,02$ & $-0,02$ & $-0,01$ & 0,759 \\
\hline \multirow[t]{3}{*}{ Lap 1} & 1 & 0,03 & $-0,14$ & $0,20 *$ & $-0,03$ & $-0,26 *$ & 0,078 \\
\hline & 2 & 0,04 & $-0,17$ & 0,08 & $-0,05$ & $-0,12$ & 0,615 \\
\hline & 3 & $0,27 * *$ & 0,13 & $-0,17$ & $-0,21$ & $-0,23 *$ & 0,010 \\
\hline Pgi 1 & 1 & $0,15^{*}$ & $-0,22 *$ & 0,09 & $-0,17$ & $-0,05$ & 0,208 \\
\hline $6 \mathrm{Pgdh} 1$ & 1 & $-0,03$ & $-0,05$ & $-0,06$ & $-0,05$ & $-0,03$ & 1,000 \\
\hline Pgm1 & 1 & $-0,16$ & 0,02 & $-0,05$ & 0,03 & $-0,05$ & 0,741 \\
\hline Média & & 0,09 & $-0,10$ & $-0,03$ & $-0,05$ & $-0,11$ & \\
\hline
\end{tabular}

$*: P \leq 5 \%$ e $* *: P \leq 1 \%$.

$P$ : Significância total do correlograma (aproximação de Bonferroni).

Tabela 3 - Índices $I$ de Moran para classes de distâncias na população PFA de $M$. urundeuva

Table 3 - Indexes of Moran I for classes of the distances in PFA population of Myracrodruon urundeuva

\begin{tabular}{|c|c|c|c|c|c|c|c|}
\hline \multirow[b]{2}{*}{ Loco } & \multirow[b]{2}{*}{ Alelo } & \multicolumn{5}{|c|}{ Classes de Distância (Número de Pares) } & \multirow[b]{2}{*}{$P$} \\
\hline & & $4 \mathrm{~m}(87)$ & $9 \mathrm{~m}(87)$ & $18 \mathrm{~m} \mathrm{(87)}$ & $78 \mathrm{~m}(87)$ & $223 \mathrm{~m}(87)$ & \\
\hline Aat 1 & 1 & $-0,13$ & $-0,01$ & $-0,04$ & 0,05 & $-0,05$ & 0,350 \\
\hline Aat 2 & 1 & $-0,02$ & $-0,13$ & 0,05 & $-0,07$ & 0,00 & 0,592 \\
\hline \multirow[t]{3}{*}{ Lap 1} & 1 & 0,03 & $-0,20^{*}$ & $-0,00$ & $-0,01$ & $-0,00$ & 0,136 \\
\hline & 2 & $-0,09$ & $-0,07$ & 0,02 & $-0,02$ & $-0,00$ & 1,000 \\
\hline & 3 & $-0,06$ & $-0,10$ & 0,03 & $-0,02$ & $-0,02$ & 1,000 \\
\hline \multirow[t]{3}{*}{ Pgil } & 1 & $-0,05$ & $-0,07$ & $-0,02$ & $-0,03$ & $-0,00$ & 1,000 \\
\hline & 2 & $-0,07$ & $-0,04$ & $-0,01$ & $-0,05$ & $-0,01$ & 1,000 \\
\hline & 3 & $0,12 *$ & $-0,05$ & $-0,21 *$ & 0,03 & $-0,07$ & 0,087 \\
\hline $6 \mathrm{Pgdh} 1$ & 1 & 0,09 & $-0,04$ & $-0,09$ & $-0,15^{*}$ & 0,01 & 0,170 \\
\hline Média & & $-0,02$ & $-0,08$ & $-0,03$ & $-0,03$ & $-0,02$ & \\
\hline
\end{tabular}

$*: P \leq 5 \%$ e $* *: P \leq 1 \%$.

$P$ : Significância total do correlograma (aproximação de Bonferroni). 
SEL

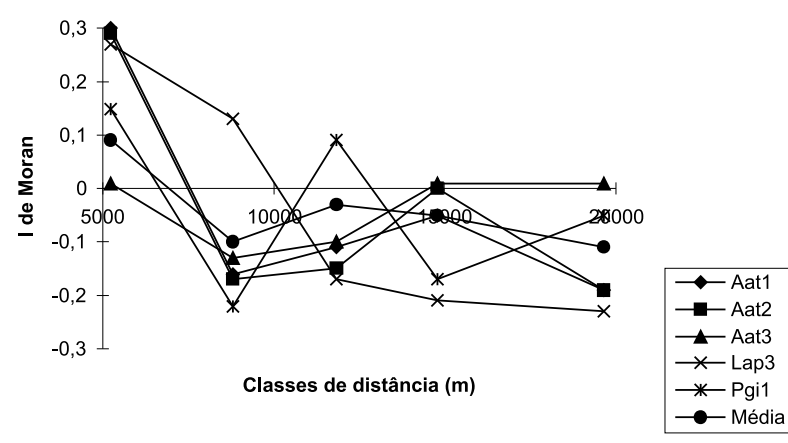

(PFA)

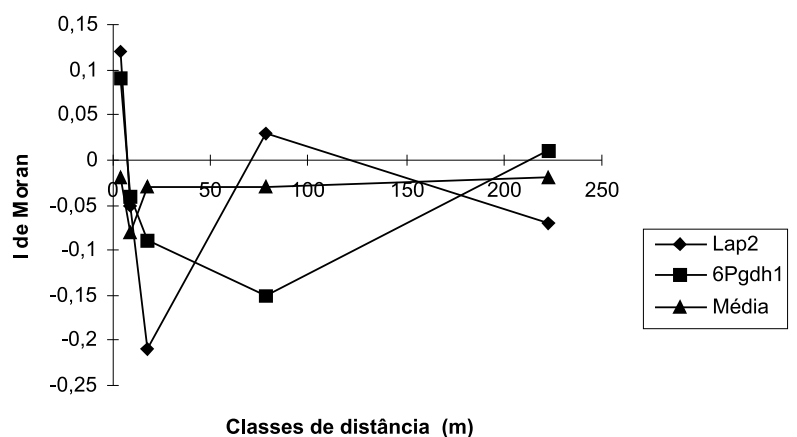

Figura 1 - Correlograma dos valores significativo e médio do índice $I$ de Moran para cinco classes de distância em populações de Myracrodruon urundeuva, localizadas em Selvíria (SEL) e Paulo de Faria (PFA).

Figure 1 - Correlagram for significant and mean values of Moran I index for five distance classes in Selvíria (SEL) and Paulo de Faria (PFA) populations.

A população SEL apresentou fortes evidências de estruturação genética espacial nas duas primeiras classes de distâncias, indicando que árvores distanciadas até aproximadamente $5.224 \mathrm{~m}$ possam ser aparentadas em algum grau e que, provavelmente, existe um limite para a dispersão de sementes na espécie. A formação de estruturação dentro de populações pode ser causada pela combinação de várias forças evolutivas, como dispersão de pólen e sementes, deriva genética, seleção para micro-"habitats" e natureza do tempo de colonização (CHUNG et al., 1999). A dispersão de sementes pelo vento em $M$. urundeuva possivelmente ocorre em maior intensidade até a distâncias de aproximadamente 5.200 $\mathrm{m}$, favorecendo a formação de manchas. A principal implicação da estruturação é o aumento da probabilidade de cruzamentos entre indivíduos aparentados, o que pode gerar endogamia na população. O parentesco entre os indivíduos corresponde à metade do coeficiente de endogamia esperado na descendência (progênies), se estes forem cruzados ou, em outros termos, a coancestria na geração parental corresponde à endogamia das progênies. Assim, quanto maior for o parentesco dentro das manchas, maior poderá ser a endogamia nas progênies.

A distância estimada para o raio da mancha (5.200) na população SEL foi maior que a relatada em outros estudos. Em Acer saccharum M. (PERRY e KNOWLES, 1991), Quercus margaretta A. (BERG e HAMRICK, 1994), Rhus trichocarpa M. (CHUNG et al., 1999), Eurya japonica T. (CHUNG e EPPERSON, 2000), Machaerium villosum V. (GIUDICE NETO e KAGEYAMA, 2000) e Pinus estrobus L. (EPPERSON; CHUNG, 2001), o raio da mancha não foi superior a $50 \mathrm{~m}$. A dispersão de sementes por anemocoria, associada à disponibilidade de espaço para o crescimento da população SEL, devido à exploração, pode ser a causa do maior tamanho da mancha em M. urundeuva. Porém, outros fatores podem estar envolvidos, como a dispersão secundária de sementes por animais.

M. urundeuva é predominantemente dióica. Portanto, autofecundações devem ser raras, e o cruzamento entre parentes deve ser a principal forma de geração de endogamia nas populações. A estruturação das populações pode favorecer a ocorrência de cruzamentos entre indivíduos aparentados e gerar endogamia. Contudo, o índice de fixação $(\hat{F})$ detectado nas progênies foi alto, mas não significativo na população SEL $(0,122)$ e alto e significativo na população PFA $(0,180)$. Provavelmente, isso esteja associado ao pequeno tamanho amostral na geração adulta e ao fato de que a amostragem só representou as árvores femininas das populações, o que poderia estar subestimando a verdadeira estruturação, devido à possibilidade de existir parentesco entre árvores masculinas e femininas. No entanto, o índice $\hat{F}$ nas árvores adultas foi negativo e significativo na população PFA $(-0,258)$ e negativo, mas não-significativo na população SEL (-0,053), indicando ausência de endogamia em ambas as populações e indícios de seleção a favor de heterozigotos na população PFA. A presença de endogamia nas progênies e a ausência e endogamia nos adultos de populações estruturadas constituem um indicativo de seleção contra homozigotos. Seleção contra homozigotos tem sido reportada em inúmeras espécies 
arbóreas, como: Pinus ponderosa L. (LINHART et al., 1981), Picea mariana (Mill.) B.S.P. (BOYLE e MORGENSTERN, 1986), Eucalyptus rhodantha B. \& S. (SAMPSON et al., 1989), Eucalyptus argutifolia G. \& B. (KENNINGTON e JAMES, 1997), Eucalyptus marginata (MILLAR et al., 2000) e Metrosideros excelsa Sol. ex Gaertn (SCHIMIDT-ADAMS et al., 2000), entre outros.

Os resultados obtidos têm fortes implicações para a conservação e o melhoramento genético da espécie. Para fins de amostragem, tanto para o melhoramento quando para a conservação genética das populações estudadas, a estruturação detectada na população SEL mostra a necessidade de se coletarem sementes em árvores distanciadas entre si por mais de $5.200 \mathrm{~m}$, para evitar coletar sementes de árvores aparentadas. A coleta de sementes em árvores distantes $100 \mathrm{~m}$ entre si tem sido preconizada para evitar árvores aparentadas, em programas de melhoramento e conservação genética (SHIMIZU et al., 1982; ELDRIDGE et al., 1993). Para a população SEL, os resultados indicam que essa estratégia pode ser ineficiente, por tratar-se de uma população com baixa densidade populacional e ter sido submetida a uma alta intensidade de exploração. Provavelmente, as matrizes amostradas sejam descendentes de poucos indivíduos remanescentes. Nesse caso, a coleta deveria ser feita em árvores espaçadas por mais de $5.000 \mathrm{~m}$. Essa prática, em populações estruturadas, evita que matrizes aparentadas sejam amostradas para os testes de progênies, mas não evita que sementes advindas dos cruzamentos entre parentes dentro das manchas sejam incluídas no teste (SEBBENN, 2002). Portanto, níveis de endogamia podem ser esperados nos testes de progênies de $M$. urundeuva, sendo estes tanto maiores quanto maior for a coancestria entre os indivíduos que darão origem às sementes. Se os níveis de endogamia forem altos, a herança de caracteres quantitativos, em programas de melhoramento, deve ser avaliada por modelos que considerem desvios das pressuposições de cruzamentos aleatórios. Em termos de conservação genética, a estruturação indica que maiores tamanhos populacionais deverão ser conservados in situ como retidos ex situ, visto que o parentesco e a endogamia reduzem tanto o tamanho efetivo de endogamia quanto o de variância.

Em vista da endogamia detectada nas populações, outra alternativa seria procurar populações sem endogamia e parentesco para a conservação in situ e coleta de sementes para programas de melhoramento, conservação ex situ e recuperação de áreas degradadas. A avaliação da estrutura genética espacial de $M$. urundeuva, em um número maior de populações e utilizando amostras maiores (pelo menos 50 indivíduos por população) do que as utilizadas no presente estudo (25 a 30), é desejável para o melhor entendimento da escala espacial da estruturação. A amostragem de 50 indivíduos adultos permitiria que número maior de classes de distâncias fosse utilizado no estudo da distribuição espacial de genótipos da espécie e, conseqüentemente, entender melhor a escada de estruturação.

\section{CONCLUSÕES}

1. Existe baixa divergência genética nas freqüências alélicas entre as populações estudadas de M. urundeuva.

2.Os níveis de variabilidade genética intrapopulacionais são altos, quando comparados com outras espécies arbóreas.

3. Existem fortes indícios de estruturação genética espacial na população mais explorada (Selvíria-MS), enquanto na população menos explorada (Paulo de Faria, SP) os indícios são de distribuição aleatória dos genótipos.

4. Os níveis de fixação de alelos são menores nas árvores adultas, comparativamente com a fase de plântulas, indicando a existência de seleção contra indivíduos endogâmicos.

\section{AGRADECIMENTOS}

Os autores são gratos à Selma Maria Bozzite de Moraes e à Elza Martins Ferraz, pelo apoio nas análises de isoenzimas; a Alexandre Marques da Silva, Gelson Dias Fernandes, Ailton dos Reis, Manoel Fernandes da Rocha Bonfim e José Cambuim, pela assistência no trabalho de campo; à Fundação de Amparo à Pesquisa do Estado de São Paulo (FAPESP), pelo financiamento do projeto; ao Conselho Nacional de Desenvolvimento Científico e Tecnológico (CNPq), pelas bolsas concedidas; e aos três revisores anônimos, pelas correções e sugestões no manuscrito.

\section{REFERÊNCIAS BIBLIOGRÁFICAS}

ALFENAS, S.A. Eletroforese de isoenzimas e proteínas afins: fundamentos e aplicações em plantas e microrganismos. Viçosa, MG: Universidade Federal de Viçosa, 1998. 574 p.

R. Árvore, Viçosa-MG, v.29, n.2, p.281-289, 2005 
BERG, E.E.; HAMRICK, J.L. Spatial and genetic structure of two sandhills oaks: Quercus laevi and Quercus margaretta (Fabaceae). American Journal of Botany, v.81, p.7-14, 1994.

BROWN, A.H.D.; ALLARD, R.W. Estimation of mating systems in open-pollinated maize populations using isozymes polymorphisms. Genetics, v.66, p.133-145, 1970.

BOYLE, T.J.B.; MORGENSTERN, E.K. Estimation of outcrossing rates in six populations of Black Spruce in central New Brunswick. Silvae Genetica, v.35, p.102-106, 1986.

CARVALHO, P.E.R. Espécies florestais brasileiras: recomendações silviculturais, potencialidades e uso da madeira. Brasília: EMBRAPA-CNPF, 1994. 640 p.

CHUNG, M.G.; CHUNG, J.M.; EPPERSON, B.K. Spatial structure of allozyme polymorphisms within populations of Rhus trichocarpa

(Anacardiaceae). Silvae Genetica, v.48, p.223227, 1999.

CHUNG, M.G.; EPPERSON, B.K. Spatial structure of allozyme polymorphisms in a population of Eurya japonica (Theaceae). Silvae Genetica, v.49, p.1-4, 2000.

CLIFF, A.D.; ORD, J.K. Spatial processes methods and applications. London: Pion Press, 1981.

COLES, J.F.; FOWLER, D.P. Inbreeding in neighboring trees in two White Spruce populations. Silvae Genetica, v.25, n.1, p.2934, 1976 .

ELDRIDGE, K. et al. Eucalypt

domestication and breeding. Oxford: Clarendon Press, 1993. 288 p.

EPPERSON, B.K. Recent advances in correlation analysis of spatial patterns of genetic variation. Evolutionary Biology, v.27, p.95-155, 1993.

EPPERSON, B.K.; CHUNG, M.G. Spatial genetic structure of allozyme polymorphisms within populations of Pinus strobus (Pinaceae).

American Journal of Botany, v.88, n.6, p.1006-1010, 2001.

R. Árvore, Viçosa-MG, v.29, n.2, p.281-289, 2005
GIUDICE NETO, J.D.; KAGEYAMA, P.Y. Estrutura genética espacial em populações naturais de Machaerium villosum Vog. (Leguminosae) na região de Moji-Guaçu, SP, Brasil. Revista Brasileira de Botânica, v.23, n.2, p.207-215, 2000.

HAMRICK, J.L.; LOVELESS, M.D. The influence of seed dispersal mechanisms on the genetic structure of plant populations. In: ESTRADA, A.; FLEMING, T.H. (Eds.) Frugivores and seed dispersal. Athens: Junk Publishers, 1986. p.124-145.

HAMRICK, J.L.; GODT, M.J.W. Allozyme diversity in plant species. In: BROWN, A.H.D. et al. (Eds) Plant population genetics, breeding and genetic resources. Sinauer: 1990. p.43-63.

KENNINGTON, W.J.; JAMES, S.H. The effect of small population size on the mating system of a rare mallee, Eucalyptus argutifolia (Myrtaceae). Heredity, v.78, p.252-260, 1997.

LACERDA, C.M.B.; KAGEYAMA, P.Y. Estrutura genética espacial de duas populações nativas de Myracrodruon urundeuva $\mathrm{M}$. Alemão na região semi-árida, Brasil. Revista Árvore, v.27, n.2, p.145-150, 2003.

LEWIS, P.0.; ZAYKIN, D. GDA - Genetic data analysis: Version 1.1 for Windows95/NT. Alburquerque: University of New Mexico. Free program distributed by the authors form <http:// www.lewis.eeb.uconn.edu/lewishome/.> 1999.

LINHART, Y.B. et al. Genetic variation in space and time in a population of ponderosa pine. Heredity, v.46, p.407-426, 1981.

MILLAR, M.A. et al. Mating system studies in jarrah, Eucalyptus marginata (Myrtaceae). Australian Journal of Botany, v.48, p.475-479, 2000.

MORAES, M.L.T. Variabilidade genética por isoenzimas e caracteres quantitativos em duas populações naturais de aroeira Myracrodruon urundeuva F.F. \& Allemão - Anacardiaceae (Syn: Astronium urundeuva (Fr. Allemão) Engler). 1992. 139f. Tese (Doutorado em Agronomia) - Escola Superior de Agricultura Luiz de Queiroz, Piracicaba, 1992. 
NOGUEIRA, J.C.B. et al. Conservação genética de essências nativas através de ensaios de progênie/ procedências. Silvicultura em São Paulo, v.16A, p.957-969, 1982.

PERRY, D. J.; KNOWLES, P. Spatial genetic structure within three Sugar Maple (Acer saccharun Marsh.) stands. Heredity, v.66, p.137-142, 1991.

RITLAND, K. Multilocus mating system program MLTR. Version 1.1. Toronto: University of Toronto, 1997.

SAKAI, A.K.; ODEN, N.L. Spatial pattern of sex expression in silver maple (Acer saccharium L.): Morista's index and spatial autocorrelation.

American Naturalist, v.122, p.489-508, 1983.

SAMPSON, J.F.; HOPPER, S.D.; JAMES, S.H. The mating system and population structure in a birdpollinated mallee, Eucalyptus rhodantha.

Heredity, v.63, p.383-393, 1989.

\section{SANTIN, D.A.; LEITÃO FILHO, H.F.}

Restabelecimento e revisão taxonômica do gênero Myracrodruon Freire Alemão (Anacardiaceae).

Revista Brasileira de Botânica, v.14, p.133-145, 1991.

SCHIMIDT-ADAMS, G.; YOUNG, A.G.; MURRAY, B.G. Low outcrossing rates and shift in pollinators in New Zealand Pohutukawa (Matrosideros excelsa; Myrtaceae). American Journal of Botany, v.87, n.9, p.1265-1267, 2000.

SEBBENN, A.M.; KAGEYAMA, P.Y.; VENCOSVKY, $\mathrm{R}$. Variabilidade genética, sistema reprodutivo e estrutura genética espacial em Genipa americana L. através de marcadores isoenzimáticos.

Scientia Forestalis, v.53, p.15-30, 1998.
SEBBENN, A.M. Número de árvores matrizes e conceitos genéticos na coleta de sementes para reflorestamentos com espécies nativas.

Revista do Instituto Florestal, v.14, n.2, p.115-132, 2002.

SHIMIZU, J.Y.; KAGEYAMA, P.Y.; HIGA, A.R. Procedimentos e recomendações para estudos com progênies de essências florestais. Colombo: EMBRAPA-URPFCS, 1982. 34p. Documento 11.

SOKAL, R.R.; ODEN, N.L. Spatial autocorrelation in biology. I methodology. Biological Journal Linnean Society, v.10, p.199-228, 1978.

SWOFFORD, D.L.; SELANDER, R.B. BIOSYS-1. A FORTRAN computer program for the analysis of allelic variation in population genetics and biochemical systematics. Journal of Heredity, v.72, p.282-283, 1989.

WARTENBERG, D. SAAP4.3. Spatial autocorrelation analyses program. Department of Environmental and Community Medicine. Robert Wood Johnson Medical School 675. Hoes Lane, Piscataway, NJ 08854. 1989.

WEIR, B.S. Genetic data analysis II. Methods for discrete population genetic data. Suderland: North Carolina State University, 1996. 445p.

WENDEL, J.F.; WEEDEN, N.F. Visualization and Interpretation of Plant Isozymes. In: SOLTIS, D.E.; SOLTIS, P.S. (Eds.). Isozymes in plant biology. Portland: Dioscorides Press, 1989. p.545, 1989. 